\title{
PARAMETER DETERMINATION OF A FUZZY LOGIC-BASED FRAMEWORK
}

\author{
Edit TÓTH-LAUFER \\ Óbuda University, Bánki Donát Faculty of Mechanical and Safety Engineering, Institute of Mechatronics \\ and Vehicle Engineering, Budapest, Hungary, laufer.edit@bgk.uni-obuda.hu
}

\begin{abstract}
During patient monitoring, personalization is extremely important in order to achieve realistic results when determining the risk level based on the measurement of physiological characteristics. This requirement can be met by a system where neither the inputs nor their number are fixed but can be defined in a personalized way. In this article, that kind of flexible framework system is presented, with particular reference to its input factors and their measurement possibilities.
\end{abstract}

Keywords: sfuzzy inference system, patient monitoring, personalization.

\section{Introduction}

Nowadays, mainly due to the aging population, there is an increasing need for patient monitoring systems. Many elderly people with chronic illnesses spend their days alone, without supervision, with a properly designed system that can save lives. As a result of technological advances, the devices are also ensured. Regrettable athlete tragedies have also highlighted that although the sport has a number of beneficial effects on the body, it may in some cases have harmful effects. There are a number of different measuring instruments available to assist in the operation of such a risk assessment system providing flexible inputs. Flexibility, in this case, means that neither the inputs themselves nor the number of the inputs are generally recorded, since the factors that need to be measured depend to a great extent on personal factors and the mode of motion chosen. During the evaluation, due to the nature of the inputs, the application of soft computing methods is justified, as there is no sharp limit for physiological characteristics [1]. In this paper, a fuzzy logic-based risk assessment framework is presented with emphasis on selecting inputs and measuring instruments.

\section{The framework}

The modular structure of the framework is applied as shown in Figure 1. Each subsystem can be considered as a fuzzy inference system, which can be modified or enlarged separately. From the study point of view, the first subsystem is the most important, which evaluates the measured values of physiological characteristics. Here, the inputs and the number of them can be personalized according to the patient's history, medical recommendations and the chosen motion form [2].

The subsystem "Activity" evaluates the characteristics of motion, taking into account its intensity, duration and frequency. The group "Environmental Factors" is important for outdoor activity, since the temperature sensation is influenced by wind, and air humidity, so their combined effect on temperature can be taken into account.

In each subsystem, a Mamdani-type inference system performs the evaluation, in which IF condition is THEN consequence-shaped natural language rules are applied. 


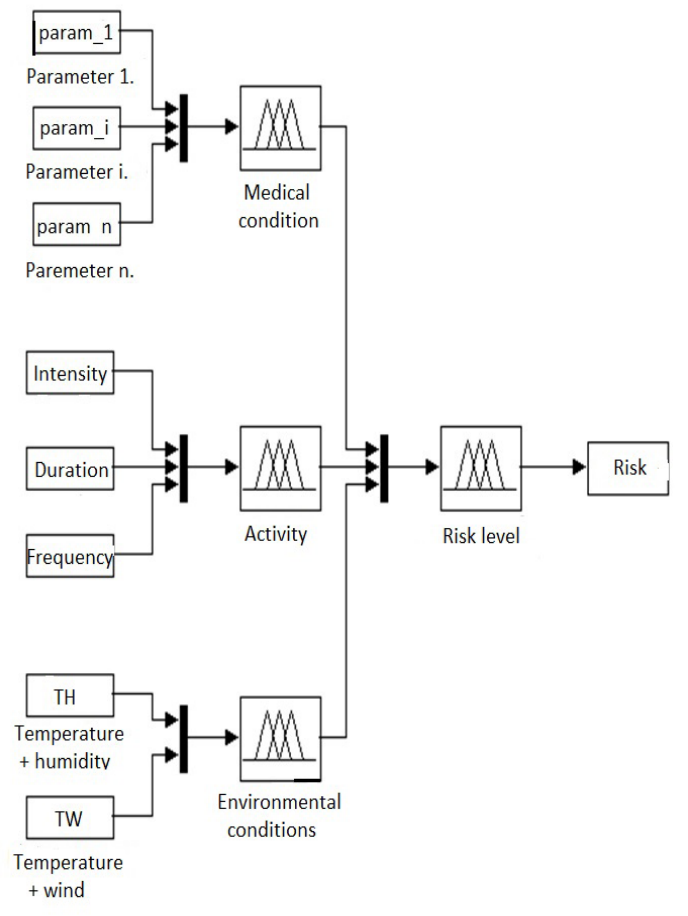

Figure 1. The system structure

\section{Inputs determination}

In defining the inputs, as mentioned earlier, personal attributes are taken into account. In order to assist this, the patient's data is stored in a personal profile, including the parameters that need to be monitored for each motion form. Inputs may be different for different patients in the same sporting activity, or, in the same patient different modes of motion can justify monitoring other factors, as illustrated in Table 1.

When determining the inputs (the physiological characteristics to be measured), the personal profile, patient history and medical recommendations are taken into account or can be influenced by the available devices. The essential, and therefore, the most commonly used input is the heart rate but blood pressure and the number of breaths are also of great importance. It is also important to note that the evaluation is done in real time, so it is necessary to weigh the fact of whether the measurement of additional input factors gives us as much additional information so it's worth affording a slower evaluation and to use devices that may be a constraint for the user, or not.
Table 1. Possible input combinations for a 28-yearold male patient (HR-heart rate, RR-respiration rate, $S B P$-systolic blood pressure, DBP-dyastolic blood pressure)

\begin{tabular}{|c|c|}
\hline Sport activity & Inputs \\
\hline Running & HR, RR \\
\hline Cycling & HR, SBP, DBP \\
\hline
\end{tabular}

\section{Measuring device selection}

When selecting the measuring devices, it is important to provide a natural environment, so as to not to interfere with the user while doing the activity, therefore it is essential to use wireless technology and it is important that do not use invasive intervention. The natural environment is not only important in terms of comfort but in some cases, it may even affect the measurement result, if the usage of the device causes stress for the patient, resulting in a false, higher measured value, rendering the evaluation unreliable. In this paper, the selection aspects of the heart rate and blood pressure measurement devices are introduced.

The heart rate measurement can be done on different principles. The simplest but at the same time the least reliable value is obtained if the pulse on the wrist is measured, which senses the pulsation of the blood vessels as a mechanical stimulus. The more modern solution is the optical pulse measurement when wearing the sensor around the forearm or upper arm and it calculates the heart rate using an algorithm based the capillary expansion while the LED illuminates the skin. However, if rapid pulse changes are to be expected, this method cannot be used because it can follow the change with difficulty and slowly, so it is not safe to use for risk assessment where filtering sudden changes is very important. Such a device is the Polar $\mathrm{OH} 1$ optical heart rate sensor.

The most reliable method is when electrodes in a chest transmitter detect the electric pulse generated by the contraction of the heart muscle and transmit it to the program, thus providing an ECG precision measurement [3]. There are several heart rate monitors that use this technology, including Polar, TomTom Runner, and Brighton Rider. For this research, the Polar V800 was chosen which can be used even during swimming thanks to its water resistance. Data is transmitted via Bluetooth to the computer or mobile phone. Automatic blood pressure measuring devices operate in such a way that a cuff, wrapped around the patient's arm is inflated and the pressure of 
the brachial artery, or in some cases of the radial artery, is measured by a sensor during deflation. The systolic and diastolic blood pressures are obtained from the measured pressure waveform, with a company-dependent mathematical algorithm, which determines the accuracy of the device [4]. For example, the blood pressure monitors, Omron M7 measuring on the upper arm, or iHealth BP7 measuring on the wrist, can be connected to a mobile phone.

\section{The evaluation process}

During the evaluation, first the measured input values must be fuzzified, i.e., the degree of belonging to each set must be given for each input belonging to the current subsystem. In the case of a heart rate, membership functions for a 37-yearold female patient are shown in Figure 2., which can be defined by (1).

$$
A_{i}(x)=\left\{\begin{array}{cc}
0 & x \leq a_{i} \\
\frac{x-a_{i}}{b_{i}-a_{i}} & a_{i} \leq x \leq b_{i} \\
1 & b_{i} \leq x \leq c_{i} \\
\frac{d_{i}-x}{d_{i}-c_{i}} & c_{i} \leq x \leq d_{i} \\
0 & d_{i} \leq x
\end{array}\right.
$$

where $a_{i}, b_{i}, c_{i}, d_{i}$ is the membership function parameters, by changing these, the functions can be shifted to the desired direction according to the user's capabilities. Measurement uncertainties and inaccuracies are also treated here by defining the parameters as the slope, support and core of the function can be controlled.

Then the firing strength is determined, where the fuzzy values are aggregated, usually by using a conjunction operator. In this case, the product operator is used (2).

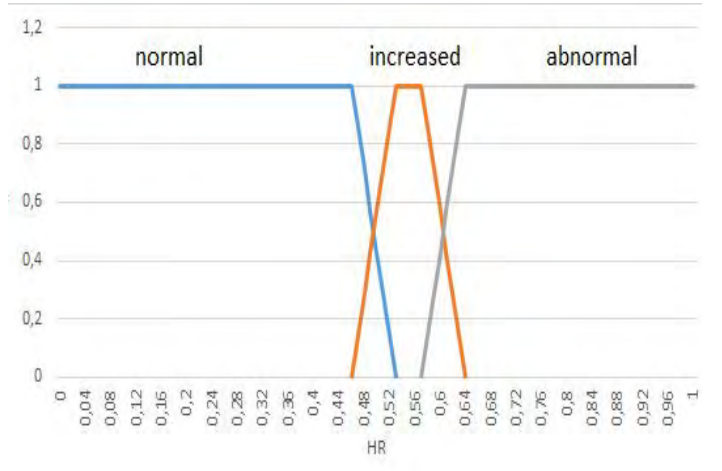

Figure 2. Input membership functions

$$
w_{i}=\prod_{j=1}^{m} \mu_{j}(x)
$$

where $\mathrm{m}$ is the number of inputs in the subsystem since combinations of the fuzzy sets of each input can be used to generate the rule premises.

The values obtained are used in the Mamdani-implication in order to determine the extent to which the rule applies to the final conclusion. The following relationship is applied to the firing strength and the consequent set belonging to the given rule:

$$
y_{Y_{i}}=w_{i} \mu_{Y_{i}}
$$

As a next step, the resulting sets are defuzzified by rules (4), and then these discrete values are aggregated (5) to generate the overall risk level for the given subsystem.

$$
\begin{gathered}
f_{i}=\frac{\int_{\sup \mu_{D_{i}}} \mu_{D_{i}}(y) y d y}{\int_{\sup \mu_{D_{i}}} \mu_{D_{i}}(y) d y} \\
O=\sum_{i=1}^{n} \bar{w}_{i} f_{i}=\frac{\sum_{i=1}^{n} w_{i} f_{i}}{\sum_{i=1}^{n} w_{i}}
\end{gathered}
$$

\section{Conclusions}

For patient monitoring systems, personalization is a basic requirement. Reliable results can only be obtained by considering the patient's features, history, and medical recommendations. The choice of inputs should, therefore, be flexibly addressed. The author has developed a framework that can handle a different number and type of inputs during the evaluation and due to device selection; it can be applied to both a mobile phone and a computer.

\section{Acknowledgement}

Supported by the ÚNKP-17-4-I. New National Excellence Program of the Ministry of Human Capacities.

\section{References}

[1] Sieira M. C., Ricart A. O., Estrani R. S.: Blood pressure response to exercise testing. Apunts Medicina de l'Esport 45/167 (2010) 191-200.

[2] Tóth-Laufer E.: A Flexible Fuzzy Logic-based Risk Assessment Framework. Óbuda University E-Bulletin 6/1. (2016) 3-8. 
[3] Dömötör E.: Pulzuskontroll, testsúlykontroll. Carita Bt, 2005, ISBN: 963-86741-0-5.

[4] Barbé K., et al, D: Analyzing the Windkessel Model as a Potential Candidate for Correcting Oscillometric Blood-Pressure Measurement. IEEE Transactions on Instrumentation and Measuremen 61/2. (2012) 411-418.

https://doi.org/10.1109/TIM.2011.2161933 